\title{
REDES SOCIAIS E O MARKETING DE INOVAÇÕES ${ }^{\mathrm{I}}$
}

\section{SOCIAL NETWORKS AND THE MARKETING OF INNOVATIONS}

\author{
HERBERT KIMURA \\ Doutor em Administração pela Escola de Administração e Economia da \\ Fundação Getulio Vargas de São Paulo (EAESP-FGV). \\ Professor adjunto associado da Universidade Presbiteriana Mackenzie (UPM). \\ Rua Santo Arcádio, 212, Brooklin - São Paulo - SP - CEP 04707- 110 \\ E-mail: herbert.kimura@gmail.com \\ LEONARDO FERIANDO CRUZ BASSO
}

PhD em Economia pela New School for Social Research (Estados Unidos).

Professor da Universidade Presbiteriana Mackenzie (UPM).

Rua Herculano de Freitas, 291, ap. 98, Bela Vista - São Paulo - SP - CEP 01308-020

E-mail: leonardobasso@mackenzie.com.br

\section{DIÓGENES MANOEL LEIVA MARTIN}

Doutor em Administração de Empresas pela Escola de Administração e Economia da

Fundação Getulio Vargas de São Paulo (EAESP-FGV).

Professor da Universidade Presbiteriana Mackenzie (UPM).

Rua Doutor Rafael Correia, 171, ap. 3, Pompéia - São Paulo - SP - CEP 05043-050

E-mail:dmmartin@mackenzie.com.br

I Os autores agradecem o apoio do Conselho Nacional de Desenvolvimento Científico do Ministério da Ciência e Tecnologia (CNPq-MCT) para o desenvolvimento deste trabalho. Uma versão do artigo foi apresentada no Encontro de Marketing da Associação Nacional de Pós-Graduação e Pesquisa em Administração (EMA-Anpad) em 2006, tendo sido agraciada com o Prêmio Raimar Richers, de melhor trabalho do evento. 


\section{RESUMO}

O entendimento da dinâmica da adoção de novas tecnologias pelos consumidores torna-se cada vez mais importante dada a velocidade de surgimento de inovações. Para a área de marketing, novos desafios surgem, pois o avanço tecnológico pode criar diferentes perfis de usuários de produtos, implicando a necessidade de implementação de novos mecanismos de sensibilização do consumidor. Considerando o marketing boca a boca num contexto de redes sociais, este artigo procura avaliar a difusão do uso de novas tecnologias. Por meio de uma modelagem estocástica, são simuladas computacionalmente populações com graus diferentes de conexões nas redes sociais, e é estimada a evolução da proporção de usuários de uma nova tecnologia. $\mathrm{O}$ uso da modelagem estocástica permite a análise de fenômenos complexos que dificilmente poderiam ser avaliados de forma empírica, por causa da problemática de criação de constructos, de levantamento de dados, de adequação de estimativas de parâmetros à realidade de novas tecnologias e de limitação das ferramentas estatísticas. Os resultados do modelo sugerem que o fortalecimento de vínculos entre indivíduos e a implementação de programas de marketing que explorem as interações nas redes sociais constituem importantes estratégias para o aumento da velocidade de difusão de tecnologias.

\section{PALAVRAS-CHAVE}

Adoção de novas tecnologias; Redes sociais; Marketing boca a boca; Modelagem estocástica de redes sociais; Difusão tecnológica.

\section{ABSTRACT}

Understanding the dynamics of the adoption of new technologies by consumers is becoming increasingly important, given the speed at which innovations emerge. For the marketing area, new challenges arise because the technological advance may create different product-user profiles, implying the need to implement new 
mechanisms in order to touch consumers. Considering word-of-mouth marketing within a context of social networks, this paper seeks to assess the diffusion of the use of new technologies. Using stochastic modeling, populations are computersimulated with different degrees of connection in social networks, and the evolution of the proportion of users of a new technology is estimated. The use of stochastic modeling allows the analysis of complex phenomena that would be difficult to assess empirically due to the problems relating to the creation of constructs, the gathering of data, the adjustment of estimates of parameters to the realities of new technologies, and the limitations of statistical tools. The results of the model suggest that the strengthening of links between individuals, as well as the implementation of marketing programs that exploit the interactions in social networks, constitute important strategies for increasing the speed of diffusion of technologies.

\section{KEYWORDS}

Adoption of new technologies; Social networks; Word-of-mouth marketing; Stochastic modeling of social networks; Technological diffusion.

\section{INTRODUÇÃO}

O entendimento da dinâmica da adoção de novas tecnologias pelos consumidores torna-se cada vez mais importante dada a velocidade de surgimento de inovações. Para a área de marketing, novos desafios surgem na medida em que o avanço tecnológico pode criar diferentes perfis de usuários de produtos, implicando a necessidade de implementação de novos mecanismos de sensibilização do consumidor.

Ozcan (200I), por exemplo, sugere a existência de um processo de transformação de uma sociedade baseada em mercado para uma sociedade baseada em redes. Assim, a lógica de mercado, na qual agentes maximizadores de satisfação competem egoística e individualmente por recursos escassos, pode se confrontar com uma lógica de redes, na qual a decisão ou o comportamento individual envolve interdependência e coordenação entre os diversos agentes, em razão das conexões dentro da rede de relacionamentos. Nesse contexto, o marketing boca a boca pode se tornar elemento crítico para a difusão de tecnologias ou padrões tecnológicos.

Ao mesmo tempo que a evolução tecnológica exige novas abordagens para o marketing, proporciona novos canais de comunicação com os consumidores. Por 
exemplo, ferramentas como e-mail, programas de mensagens instantâneas e voiceover-IP trazem maiores oportunidades para a geração de relacionamentos e manutenção de interação entre indivíduos. Essas novas formas de comunicação, baseadas na internet, podem ter um impacto relevante na propagação de um produto por meio do marketing boca a boca, que é considerado por Goldberg et. al (200I) um fenômeno ao mesmo tempo difundido e intrigante.

Considerando o marketing boca a boca num contexto de redes sociais, este artigo procura avaliar a difusão do uso de novas tecnologias. Por meio de uma modelagem estocástica, são simuladas computacionalmente populações com graus diferentes de conexões nas redes sociais, e é estimada a evolução da proporção de usuários de uma nova tecnologia, de acordo com diferentes parâmetros. Em um ambiente em que os produtos de tecnologia têm um ciclo de vida cada vez menor, as tecnologias são rapidamente substituídas e a consolidação de padrões tecnológicos torna-se fator crítico para as empresas, é relevante identificar possíveis estratégias para o aumento da velocidade de adoção de tecnologias pelos consumidores.

O uso de um modelo teórico é apropriado, tendo em vista que a complexidade da avaliação empírica das interações dentro das redes sociais dificulta a análise de influências de variáveis individuais no processo de difusão de tecnologias. Especificamente, métodos tradicionais de análise empírica em marketing, como análise fatorial e regressão múltipla, comumente utilizam premissas de linearidade que podem não extrair relacionamentos mais complexos. Além disso, o aumento do poder computacional possibilita que comportamentos não-lineares sejam simulados diversas vezes e em diversas iterações, permitindo a geração de várias possíveis situações. Apesar de os modelos teóricos não contemplarem a riqueza de detalhes do mundo real, representam importantes instrumentos para a projeção de cenários.

Considerando o caso específico de estudo de novas tecnologias, dificilmente seria possível a estimação de comportamento futuro baseado em dados empíricos históricos, por pelo menos dois motivos: I. as condições iniciais das tecnologias passadas têm baixa probabilidade de se manterem constantes para o estudo de tecnologias futuras e 2. os mecanismos de difusão de informação e canais de marketing podem variar consideravelmente em um curto espaço de tempo. Por exemplo, blogs especializados constituem um novo canal de difusão de informação, constituindo potenciais alvos para a atuação do marketing boca a boca.

Finalmente, uma outra vantagem do modelo teórico para o estudo deste artigo está associada à possibilidade de investigação de um fenômeno sob condições controladas, uma vez que as variáveis são explicitamente definidas, não havendo incertezas sobre seu efetivo conceito. Em uma análise empírica, haveria a necessidade de operacionalização de um constructo com base em variáveis que 
poderiam não representar adequadamente um dado conceito. Por exemplo, em marketing, estudos empíricos que envolvem questionários podem ter dificuldades em identificar variáveis que reflitam com acurácia a percepção ou a opinião sobre o atributo de um produto. Além disso, as escalas das variáveis obtidas em pesquisas empíricas geralmente violam premissas importantes dos métodos estatísticos utilizados.

Neste estudo, com base no modelo estocástico, são geradas simulações para identificar o impacto de vários parâmetros na evolução da proporção de usuários de uma nova tecnologia. Após a apresentação de um breve referencial teórico sobre redes sociais e marketing boca a boca, é discutido o modelo matemático que embasa o estudo. Nesta descrição, novos referenciais são acrescentados para justificar a escolha das variáveis relevantes do modelo. Ênfase é dada na descrição da modelagem, pois as premissas utilizadas podem ter um impacto considerável nos resultados. Finalmente, são realizadas análises das simulações do modelo teórico. Os resultados do modelo sugerem que o fortalecimento de vínculos entre indivíduos e a implementação de programas de marketing que explorem as interações nas redes sociais constituem importantes estratégias para o aumento da velocidade de difusão de tecnologias.

O modelo desenvolvido pode ser usado para a projeção de cenários sobre a difusão de inovações, considerando diferentes densidades das redes sociais. Destaca-se que grande parte do artigo recai sobre a discussão da modelagem, pois a compreensão do modelo possibilita que o algoritmo possa ser aplicado na projeção de diferentes cenários sobre a evolução do uso tecnologias com várias características distintas.

\section{REFERENCIAL TEÓRICO}

A rede social é uma representação das relações e interações entre indivíduos de um grupo e possui um papel importante como meio de propagação de informação, idéias e influências (Kempe et al., 2005). Considerando essa perspectiva, o estudo do marketing boca a boca ou word-of-mouth marketing, que envolve as interações dentro da rede social, tem gerado crescente interesse, principalmente pelas possibilidades trazidas pela internet (GOLDBERG et al., 200I).

A perspectiva das redes sociais está ligada à idéia de que as interações sociais aprimoram ou restringem o acesso a recursos (BRASS, I984). Nesse contexto, neste artigo, é analisado o acesso à informação ou particularmente a adoção de determinada tecnologia, considerando não somente os aspectos de marketing tradicionais, disponibilizados por meio de meios convencionais, como também a influência da estrutura das redes sociais que servem de base para o marketing boca a boca. 
De acordo com Collier (I998), a interação social incorpora um capital social e pode gerar três externalidades: o conhecimento sobre o comportamento dos outros agentes; o conhecimento sobre o mercado, tais como preços e tecnologias; e o benefício da ação coletiva. Esse capital social, baseado em um conjunto de associações entre pessoas, consiste em interações e normas que têm efeito na produtividade de uma comunidade (PUTNAM, I993). Assim, a interação entre os indivíduos dentro de uma sociedade conduz à formação de laços ou conexões que impactam o desempenho do grupo. Para o caso específico da análise da tecnologia, as interações nas redes podem influenciar a evolução do uso de determinados produtos.

Estudos sobre marketing boca a boca e comportamento do consumidor podem ser encontrados em Eliashberg et al. (2000), Krider e Weinberg (I998), Buttle (I998) etc. De acordo com Walker (I995), 40\% dos norte-americanos procuram conselhos de amigos e familiares para obter serviços de médicos, advogados e mecânicos, demonstrando a relevância do conceito do marketing boca a boca no processo decisório. Rogers (I995) sugere que o marketing boca a boca também constitui importante aspecto para deliberações sobre a aquisição de novos produtos ou sobre o uso de inovações.

Conforme Goldberg et. al (200I), a maioria dos estudos sobre marketing boca a boca e, mais recentemente, sobre o marketing pela internet, denominado marketing viral ou word-of-mouse marketing, concentra-se no estudo individual, não fornecendo indicações práticas, num nível macro, sobre como mitigar a ineficácia de programas voltados à propagação de informação por meio das interações entre os indivíduos. Para Silverman (I997), vários administradores consideram que influências pessoais sobre a adoção de tecnologias ou aquisição de produtos não estão sob seu controle. Sob essa perspectiva, o uso efetivo ou o direcionamento adequado do marketing boca a boca seria pouco explorado.

Ardnt (1967) estabelece que o marketing boca a boca representa um tipo diferente de fenômeno de rede, pois o diálogo entre agentes torna-se intrumental tanto nas trocas entre os indivídus quanto no ajuste de oferta e demanda. Deve-se destacar a abrangência dos estudos sobre redes. Exemplificando, sob a perspectiva social, Armengol e Jackson (2004) estudam os efeitos das redes sobre desigualdade e empregabilidade. O uso político das redes sociais é estudado por Murphy e Shleifer (2004). Sob uma abordagem empresarial, Podolny e Baron (1997) sugerem que redes informais possibilitam: a troca de recursos, como experiências ou dicas sobre atividades; a difusão de informações estratégicas associadas ao trabalho; e a definição de normas e da identidade de uma empresa. Com relação à dinâmica de negócios, Rauch (200I) apresenta evidências de que as redes sociais promovem o comércio internacional, pelo fato de este reduzir problemas de execução de contratos e de difusão de informações sobre 
oportunidades de negócio. Essa difusão de informação fundamenta também a evolução do uso de tecnologias em decorrência das redes sociais.

No contexto das inovações recentes, Wejnert (2002) desenvolve uma estrutura conceitual baseada em redes sociais para explicar a difusão de tecnologias. Além disso, novas configurações de trabalho, como colaboração on-line ou a distância, podem ter um componente de rede de relacionamento que impulsiona a produtividade. Por exemplo, Herbsleb e Mockus (2003) estudam o desenvolvimento global de software, testando hipóteses que envolvem características de redes sociais distribuídas geograficamente, enquanto Leenders et al. (2003) abordam a comunicação, a virtualidade e a criatividade de equipes a partir de uma perspectiva de redes sociais.

Com o aumento da capacidade de processamento numérico propiciado pela informática, a abordagem computacional para o estudo de redes sociais tem possibilitado que sistemas mais sofisticados sejam simulados e avaliados em maiores detalhes. Harrison e Carroll (2002) estudam o comportamento de redes de influência ao longo do tempo, utilizando um algoritmo baseado em um modelo formal de transmissão cultural em organizações. Destaca-se, em termos tecnológicos, que a própria internet pode ser considerada uma rede social sem precedentes, dados sua escala e poder de influência (OTTE; ROUSSEAU, 2002).

\section{DISCUSSÃO DA MOdELAGEM MATEMÁTICA}

\subsection{CONTEXTUALIZAÇÃO}

Na modelagem, consideramos um novo produto cujo mercado potencial ou público-alvo é composto por "n" indivíduos. Inicialmente, todos os indivíduos são sadios. Ou seja, os indivíduos ainda não foram infectados e, portanto, não adquiriram o novo produto. Na prática, produtos com inovações tecnológicas substanciais são compatíveis com essa modelagem, pois, no momento inicial, não existe uma base instalada que poderia influenciar a decisão de compra.

Apesar de poder ser usado de maneira mais genérica, o modelo será explicado por meio de uma aplicação específica para a difusão do uso de tecnologia. Assim, como nos modelos seminais de Rogers (I962) e Bass (I969), o modelo deste artigo é mais apropriado para avaliar a difusão de uma tecnologia e não de uma marca específica. Por exemplo, o modelo é mais adequado para a análise de vendas genéricas de telefones celulares ou de tocadores de $\mathrm{MP}_{3}$ do que para a análise de possíveis vendas de celulares ou tocadores de $\mathrm{MP}_{3}$ de marcas específicas. 
O modelo é composto por duas fases: construção da topologia da rede social e dinâmica do contágio da tecnologia. Na primeira fase, são construídas as redes de relacionamento entre os indivíduos. As conexões dos indivíduos na rede dependem de características que são distribuídas de modo aleatório na população. Assim, os "n" indivíduos do público-alvo podem estar ligados entre si através de conexões, que serão associadas a contatos sociais. As conexões poderão ser fracas ou fortes, dependendo da proximidade das características dos indivíduos.

$\mathrm{Na}$ segunda fase, é definida matematicamente a dinâmica de contágio da tecnologia entre os diversos agentes na população. O contágio pode ocorrer de duas maneiras: fora da rede social ou dentro dela. O marketing dos produtores da tecnologia e a tendência de mercado podem transformar indivíduos inicialmente sadios, que em um instante não estão informados sobre uma determinada tecnologia ou não estão ainda convencidos sobre os benefícios de um produto, em indivíduos infectados, ou seja, usuários da tecnologia. Considera-se que esse tipo de contágio ocorre em um ambiente externo à rede social.

Além disso, uma vez que estejam utilizando uma tecnologia, os indivíduos infectados podem contaminar outros indivíduos por meio da interação na rede social. Assim, indivíduos inicialmente sadios, ao interagirem com aqueles infectados, também podem se contaminar e comprar o novo produto. Por exemplo, um indivíduo pode, ao interagir com um amigo que usa Linux, ser sensibilizado, convencer-se dos benefícios do produto e tornar-se também um usuário. Esse tipo de contágio ocorre, portanto, em um contexto interno à rede social.

A seguir, são discutidas as duas fases da modelagem de maneira mais detalhada. Além disso, a escolha das variáveis do modelo é justificada com base em referências bibliográficas. É importante destacar, porém, que a operacionalização das variáveis pode impor barreiras à implementação prática do modelo por causa da dificuldade em determinar os constructos em uma pesquisa empírica. Em contrapartida, o uso de simulação em modelos teóricos possibilita que relações complexas sejam investigadas sem limitações de caráter empírico, como ruído no levantamento de dados, inadequação de constructos e escala inapropriada do instrumento de medição.

\subsection{FORMAÇÃO DA TOPOLOGIA DAS REDES DE RELACIONAMENTO}

No modelo, inicialmente é definida a topologia das redes sociais, ou seja, a estrutura das conexões que inter-relacionam os diversos indvíduos. A topologia será mantida constante durante o processo de propagação da tecnologia entre as conexões. Essa suposição pode ser violada na prática, uma vez que constantemente as pessoas interagem com outras, podendo formar vínculos novos. Porém, 
nos casos em que a velocidade de difusão da tecnologia é razoavelmente grande e a velocidade de formação de vínculos é baixa, a suposição não deve ter impacto relevante. Obviamente, como decorrem de um modelo com diversas premissas, os resultados devem ser vistos com cautela.

A formação da rede de relacionamento de cada indivíduo depende de duas características em comum, que são tratadas de maneira genérica no modelo como: cultura e gosto.

\subsubsection{Características culturais}

Simplificadamente, o modelo supõe que as redes de relacionamento do indivíduo estão associadas à difusão de aspectos culturais como Axelrod (I997) propõe. Os aspectos ligados à cultura são tratados neste artigo de modo bastante genérico. Dado o foco deste estudo, não será discutido o conceito de cultura, uma vez que é extremamente extenso e complexo. Kroeber e Kluckhohn (I952), por exemplo, apresentam uma lista de mais de i6o definições para o termo cultura.

Neste artigo, de maneira simples, aspectos culturais são compostos por atributos como origem familiar, origem étnica, nível socioeconômico etc. Portanto, usa-se a definição, comumente determinada como cultura histórica, associada a heranças sociais que adquirem um significado social em razão de uma origem racial e história de vida de um grupo (PARK; BURGESS, I92I).

No modelo, supõe-se que quanto mais próximas as características culturais dos indivíduos, maior a probabilidade de compartilharem uma conexão em uma rede social. Ou seja, as conexões nas redes de relacionamento são mais prováveis entre indivíduos da mesma família, de mesmos valores, da mesma escola, do mesmo círculo social etc. Para cada indivíduo i, atribui-se aletaoriamente, de acordo com uma distribuição normal padronizada, um valor CULT(i).

\subsubsection{Características de gosto ou preferência}

Conforme sugere Bourdieu (I984), o gosto classifica um bem ou produto e também o classificador. Os indivíduos sociais são classificados pelas classificações que realizam, são distinguidos pelas distinções que realizam entre o bonito e o feio, entre o que é distinto e o que é vulgar etc. (BOURDIEU, I984). Liu et al. (2005), ao estudarem redes de relacionamento on-line, identificaram padrões profundos de cultura e gosto, que sobrepujam os aspectos superficiais de estrutura de conectividade social. Dessa maneira, o gosto pode ter um papel relevante dentro da estrutura das redes sociais. No modelo, a cada indivíduo i é atribuído um valor TASTE(i), selecionado de uma distribuição uniforme, para a característica de gosto. 
Assim, apesar de aspectos culturais serem importantes para formação de conexões nas redes de relacionamento, gostos ou preferências semelhantes podem também constituir importantes formadores de conexões. Exemplificando, pessoas com origens culturais diferentes podem ter gostos musicais semelhantes. Nesse caso, quanto mais próximos os gostos músicais dos indivíduos, maior a probabilidade de formação de conexão, uma vez que esses indivíduos podem freqüentar os mesmos shows ou acessar os mesmos fóruns on-line.

Tendo sido estabelecidos os valores das variáveis CULT e TASTE para cada indivíduo, o modelo identifica proximidades de cultura e de gosto para indivíduos, dois a dois. As proximidades de cultura ProxCULT $(i, j)$ e de gosto ProxTASTE(i,j) de dois indivíduos i e j são dadas, respectivamente, por:

$$
\begin{aligned}
& \operatorname{ProxCULT}(\mathrm{i}, \mathrm{j})=1-\frac{|\operatorname{CULT}(\mathrm{i})-\operatorname{CULT}(\mathrm{j})|}{\max _{\mathrm{k}=1, \ldots, \mathrm{N}}(\operatorname{CULT}(\mathrm{k}))-\min _{\mathrm{k}=1, \ldots, \mathrm{N}}(\operatorname{CULT}(\mathrm{k}))} \\
& \operatorname{ProxTASTE}(\mathrm{i}, \mathrm{j})=1-\frac{|\operatorname{TASTE}(\mathrm{i})-\operatorname{TASTE}(\mathrm{j})|}{\max _{\mathrm{k}=1, \ldots, \mathrm{N}}(\operatorname{TASTE}(\mathrm{k}))-\min _{\mathrm{k}=1, \ldots, \mathrm{N}}(\operatorname{TASTE}(\mathrm{k}))}
\end{aligned}
$$

Ou seja, a proximidade entre individíduos, em razão de seus traços culturais ou de gosto, é dada por um número que varia de o a I. Esse intervalo unitário decorre da divisão da diferença absoluta entre os valores das variáveis entre os indivíduos i e j, pela diferença entre os valores máximos e mínimos das variáveis em toda a população. Nas equações anteriores, quanto menor a diferença entre os valores de CULT ou TASTE entre os indivíduos, maior a proximidade cultural ou de gosto.

A possível conexão entre indivíduos decorre da proximidade conjunta, levando-se em consideração tanto aspectos culturais quanto as características de gosto. Essa proximidade na rede tem reflexo na força da conexão, denotada pela variável LinkSTRENG. Para incorporar um parâmetro que mensura a proximidade conjunta, é realizada uma soma vetorial de ProxCULT e ProxTASTE. Tendo em vista que existe uma possibilidade de a cultura ter relação com gosto, o modelo ainda incorpora um coeficiente de correlação $\rho$ entre as proximidades. Por exemplo, dois indivíduos podem gostar de tipos de comida semelhantes por causa de uma origem étnica comum, ou seja, em razão de características culturais.

A Figura I mostra a forma de obtenção da força da conexão LinkSTRENG(i,j) entre dois indivíduos, a partir de ProxCULT $(i, j)$ e ProxTASTE(i,j). Assim, na modelagem utiliza-se a relação entre aspectos culturais e de gosto refletida no co-seno do ângulo entre as dimensões de proximidade cultural e de proximidade de gosto. 


\section{FIGURA I}

OBTENÇÃO DA FORÇA DA CONEXÃO ENTRE DOIS INDIVÍDUOS

EM RAZÃO DAS RELAÇÕES ENTRE AS PROXIMIDADES DE

CULTURA E DE GOSTO PARA: (A) $\rho=0$; (B) $\rho>0$ E (C) $\rho<0$

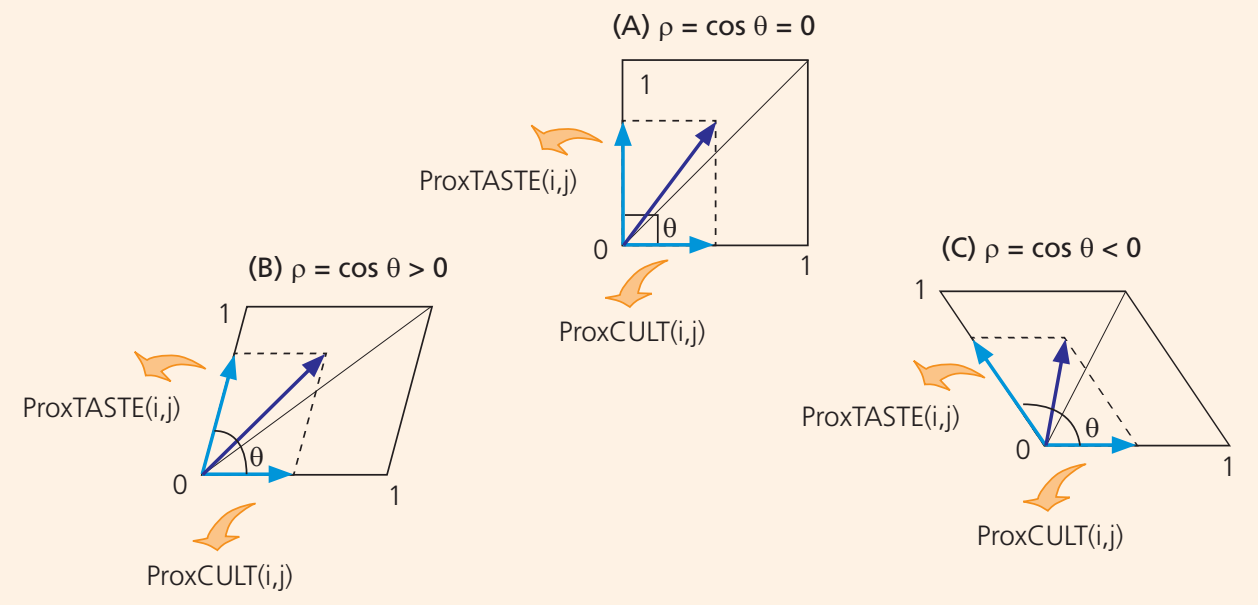

Matematicamente, com a finalidade de padronização para o intervalo [o,I], a proximidade na rede ou força da conexão é definida como:

$\operatorname{LinkSTREnG}(i, j)=\frac{\sqrt{\operatorname{ProxCULT}(i, j)^{2}+\operatorname{ProxTASTE}(i, j)^{2}+2 \cdot \operatorname{ProxCULT}(i, j) \cdot \operatorname{ProxCULT}(i, j) \cdot \rho}}{\sqrt{2(1+\rho)}}$

Conforme sugere Granovetter (I973), as interações nas redes sociais envolvem não somente conexões fortes, mas também conexões fracas. A distinção entre os tipos de conexão é apresentada na Figura 2.

\section{FIgURA 2}

STATUS DA CONEXÃO EM RAZÃO DA FORÇA DA CONEXÃO

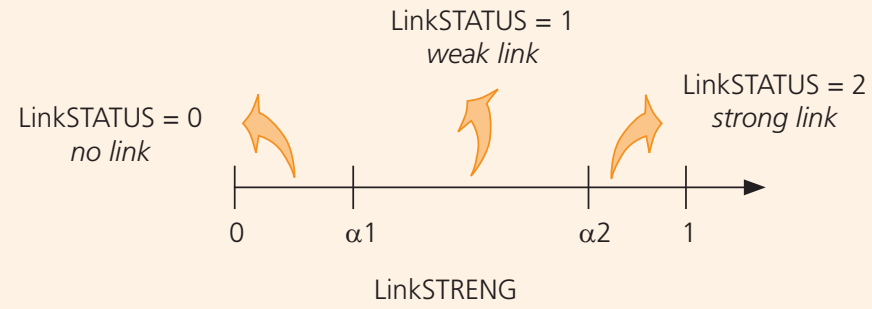


Para cada par de indivíduos, definem-se limites mínimos de LinkSTRENG para que sejam estabelecidas conexões fracas e fortes. Em termos gerais, dados os parâmetros de proximidade $\alpha_{\mathrm{I}}$ e $\alpha_{2}$, que representam respectivamente a força mínima necessária para que as conexões sejam fracas ou fortes, as seguintes condições valem:

$$
\operatorname{LinkSTATUS}(i, j)=\left\{\begin{array}{l}
0, \text { if } 0 \leq \operatorname{LinkSTRENG}(i, j)<\alpha_{1} \\
1, \text { if } \alpha_{1} \leq \operatorname{LinkSTRENG}(i, j)<\alpha_{2} \\
2, \text { if } \alpha_{2} \leq \operatorname{LinkSTRENG}(i, j)<1
\end{array}\right\}
$$

onde:

- $\quad \alpha_{1}$ representa o limite mínimo de LinkSTRENG para que a conexão seja considerada fraca;

- $\quad \alpha_{2}$ representa o limite mínimo de LinkSTRENG para que a conexão seja considerada forte;

- LinkSTATUS representa o status da conexão entre i e j.

\subsection{DINÂMICA DO CONTÁGIO}

Uma vez construída a estrutura de conexões da rede social, passa-se à modelagem da dinâmica do contágio da tecnologia entre os indivíduos da população. Conforme já apresentado, indivíduos podem ser infectados pela tecnologia de duas maneiras: de maneira externa à rede social e de maneira interna a ela.

$\mathrm{Na}$ perspectiva externa à rede social, os indivíduos podem ser infectados de duas formas: pela propensão pessoal para adoção de uma determinada tecnologia e pela evolução da tecnologia na população. Esses mecanismos de contágio estão associados ao que Bass (1969) classifica de influências externas, que, por sua vez, definem um coeficiente de inovação. No modelo de Bass (1969), esse coeficiente de inovação define a probabilidade de que indivíduos sadios passem a adotar uma tecnologia em razão da cobertura da mídia e de outros fatores externos.

\subsubsection{Propensão na adoção de tecnologia}

No momento inicial, tendo em vista que não existe nenhum indivíduo infectado pela tecnologia, o processo de difusão não ocorre através do contágio. Os primeiros usuários da tecnologia, de acordo com Rogers (I995), seriam os agentes inovadores. No modelo, esses agentes inovadores somente surgiriam em decorrência dos esforços de marketing dos produtores da tecnologia. Assim, os inovadores, com status inicial de sadios, passariam para o status de infectados, ao serem sensibilizados pelas mensagens de marketing tradicionais, como por meio de comerciais ou propagandas em televisão, rádio e jornais. 
Cada indivíduo possui uma característica associada à propensão em ser contaminado por determinada tecnologia, por causa da cobertura da mídia ou dos esforços de marketing fora da rede de relacionamentos. A cada um dos indivíduos é atribuído um valor para essa propensão WILLINGNESS, selecionado por meio de um sorteio aleatório de uma distribuição uniforme no intervalo [o,I]. Deve-se ressaltar que a propensão representa variável relevante para o estudo de uso de tecnologias. Por exemplo, Rogers (I995) estabelece que os inovadores, em geral, têm uma maior propensão a assumir riscos. Considerando inovações na área de medicina, Barton e McManus (2005), em pesquisa sobre tecnologias para tratamento de infertilidade, obttiveram resultados que sugerem que fatores gerais, entre eles a propensão à adoção de novas tecnologias, têm relação com o uso de produtos ou processos inovadores.

A probabilidade de um indivíduo sadio i tornar-se infectado pelo marketing tradicional depende de uma probabilidade-base $\beta$ e de sua propensão WILLINGNESS(i) a adotar inovações. Indivíduos com maior interesse em produtos eletrônicos, por exemplo, devem ter maior probabilidade de se infectar. Em contrapartida, indivíduos que não possuem facilidade em lidar com inovações têm menor probabilidade de se infectar. Para fins de modelagem, consideramos uma relação linear entre a probabilidade de infecção via os métodos tradicionais de marketing e a propensão do indivíduo, ou seja:

$$
\operatorname{ProbMARK}(\mathrm{i})=\beta . \text { WILLINGNESS }(\mathrm{i})
$$

onde: $\beta$ representa o esforço de marketing, no intervalo [o,I], por meio de cobertura externa à rede.

\subsubsection{Evolução da tecnologia na população}

Além de os esforços de marketing constituírem importantes componentes exógenos à rede para o contágio de indivíduos, outro aspecto relevante está associado à evolução da tecnologia na população. À medida que indivíduos tornam-se infectados, a nova tecnologia começa a ganhar força e novos indivíduos tornamse usuários, simplesmente pela tendência de mercado, independentemente da influência dos esforços de marketing tradicionais.

Por exemplo, a internet pode ter parte de seu crescimento associada à evolução intrínseca da tecnologia e dos meios de comunicação. Quanto mais usuários se conectam à internet, maior será a probabilidade de outros usuários também a usarem. Não há necessariamente um marketing específico da internet. Porém, existe uma tendência, imposta por pressões exógenas, que aumenta o número de usuários. Se mais pessoas se conectam on-line via programas de mensagens ins- 
tantâneas, mais pessoas sentem-se atraídas ou até mesmo forçadas a usar essa tecnologia. Além disso, os custos para o cliente podem diminuir, por causa do ganho de escala e do maior domínio da tecnologia por parte dos fornecedores.

No modelo, existe uma probabilidade de contaminação de um indivíduo sadio que depende do número atual de usuários. Considerando um modelo de difusão semelhante ao de Bass (I969), pode-se estabelecer uma modelagem na qual a probabilidade de infecção aumenta com o número de indivíduos contaminados. Porém, em vez de se considerar uma função de probabilidade convexa nos estágios iniciais de difusão da tecnologia e côncava nos estágios finais, adota-se uma função côncava em todo o intervalo. Matematicamente, para cada indivíduo i, a função de probabilidade ProbTREND(i) de adoção da tecnologia em razão da tendência de mercado, representada pela proporção de usuários, é dada por:

$$
\operatorname{ProbTREND}(\mathrm{i})=\gamma \cdot\left(1-\exp \left(-\mathrm{N}_{\text {inf ect }} / \mathrm{N}\right)\right)
$$

onde:

- $\quad \gamma$ representa uma probabilidade básica de difusão da tecnologia, independentemente da proporção de usuários;

- $\quad \mathrm{N}_{\text {inf ect }} / \mathrm{N}$ representa a proporção de usuários da tecnologia. Observe que ProbTREND é igual a todos os indivíduos.

A partir de uma perspectiva interna à rede social, a difusão da tecnologia pode ocorrer pela influência de indivíduos infectados em indivíduos sadios, por meio das conexões. No modelo de Bass (1969), essa forma de difusão está caracterizada por um parâmetro denominado coeficiente de imitação, que decorre da transmissão via o marketing boca a boca, a partir de indivíduos que já usam determinado produto.

\subsubsection{Contágio pelas conexões na rede social}

No modelo deste artigo, cada indivíduo tem uma mesma probabilidade-base $\delta_{\text {I }}$ de ser infectado, por causa das conexões fracas da sua rede de relacionamento. Além disso, cada indivíduo também possui uma probabilidade-base $\delta_{2}$ de ser infectado a partir das conexões fortes na rede. Esses mecanismos de difusão são semelhantes ao proposto por Goldberg et. al (200I) para o estudo do marketing boca a boca usando autômatos celulares. A probabilidade-base de contaminação, seja $\delta_{\text {I }}$ seja $\delta_{2}$, é ajustada pelas conexões do indivíduo sadio na rede social.

Por exemplo, ao interagir com indivíduos infectados, um indivíduo sadio apresenta um acréscimo na probabilidade de adotar a tecnologia em decorrência da troca de informação sobre a inovação. De certa maneira, o modelo simula a 
propagação de informação sobre a tecnologia em estudo. A influência do indivíduo infectado na probabilidade de um outro indivíduo adotar a tecnologia é tanto maior, quanto maior for a variável reputação. Ou seja, quanto maior a reputação de um indivíduo infectado maior a probabilidade de a interação com um indivíduo sadio resultar num novo usuário da tecnologia.

Assim, usuários que são considerados conhecedores da tecnologia possuem um peso maior na decisão de um indivíduo sadio sobre uma possível adoção da tecnologia. A escolha da reputação como variável relevante no processo de difusão da tecnologia tem fundamentação na área de economia (KREPS; WILSON, I982) e no campo da sociologia (RAUB; WEESIE, 2000): indivíduos tomam decisões ou apresentam determinados comportamentos com base na informação sobre as atitudes e reputações de outras pessoas.

Considerando os resultados empíricos apontados por Resnick e Zeckhauser (200I) e Lin et al. (2002), a cada indivíduo i é associado um valor de reputação, Reputation(i), extraído de uma distribuição de probabilidades exponencial, no intervalo de $\circ$ a I. Ou seja, segundo esse modelo, existem poucos indivíduos com reputação elevada. Em outras palavras, os experts de uma tecnologia nova são escassos. Apesar de reputação e propensão poderem ser correlacionadas, no modelo consideramos que essas variáveis são ortogonais. Em termos matemáticos, inicialmente, as probabilidades ProbWEAK e ProbSTRONG de adoção de tecnologia a partir de interações através de conexões fracas e fortes dependem da propensão do indivíduo em adotar a tecnologia e são dadas, respectivamente, por:

$\operatorname{ProbWEAK}(\mathrm{i})=\delta_{\mathrm{I}}$.WILLINGNESS(i) e ProbSTRONG $(\mathrm{i})=\delta_{2}$. WILLINGNESS $(\mathrm{i})$

As interações na rede social fazem que as probabilidades de adoção ProbWEAK e ProbSTRONG sofram alterações. Quanto maior a reputação dos indivíduos infectados j com os quais os indivíduos sadios i interagem, maiores ProbWEAK e ProbSTRONG, de acordo com o seguinte algoritmo: 


\section{FIGURA 3}

\section{ALGORITMO DA PROBABILIDADE DE CONTÁGIO ATRAVÉS DA REDE SOCIAL}

Para cada $\mathrm{j}<>\mathrm{i}$, sendo $\mathrm{j}$ um indivíduo infectado, ligado ao indivíduo sadio $\mathrm{i}$ através de conexões fracas (LinkSTATUS = I),

se houver interação entre i e j então

$\operatorname{ProbWEAK}(\mathrm{i})=\mathrm{I}-(\mathrm{I}-\operatorname{ProbWEAK}(\mathrm{i})) \cdot\left(\mathrm{I}-\delta_{\mathrm{I}} \cdot \operatorname{Reputation}(\mathrm{i})\right)$ caso contrário,

$\operatorname{ProbWEAK}(\mathrm{i})=\delta_{\mathrm{I}} \cdot$ WILLINGNESS $(\mathrm{i})$

Para cada $j<>i$, sendo $\mathrm{j}$ um indivíduo infectado, ligado ao indivíduo sadio $\mathrm{i}$ através de conexões fortes (LinkSTATUS $=2$ ),

se houver interação entre i e j então

ProbSTRONG(i) $=\mathrm{I}-(\mathrm{I}-\operatorname{ProbSTRONG}(\mathrm{i})) \cdot\left(\mathrm{I}-\delta_{2} \cdot \operatorname{Reputation}(\mathrm{i})\right)$ caso contrário,

ProbSTRONG $(\mathrm{i})=\delta_{2}$. WILLINGNESS(i)

Deve-se observar que essas igualdades representam um algoritmo iterativo, ou seja, ProbWEAK e ProbSTRONG são atualizados a cada interação de i com j, em razão das conexões fracas ou fortes da rede social, ou seja, quando LinkSTATUS(i,j) = I ou 2, respectivamente. Quando a conexão inexiste, ou seja, quando LinkSTATUS entre dois indivíduos é igual a zero, não há possibilidade de contágio, uma vez que os dois indivíduos não estão relacionados na rede social. Segundo esse modelo, portanto, elimina-se a possibilidade de contágio espúrio em razão de interações casuais entre indivíduos que não possuem nenhuma conexão através da rede social.

Além disso, a cada iteração do algoritmo, não há necessariamente uma interação entre os indivíduos. A probabilidade de uma interação ocorrer em cada iteração do algoritmo entre indivíduos ligados por conexões fracas é $\varepsilon_{\mathrm{I}}$ e a probabilidade de uma interação ocorrer em cada iteração entre indivíduos ligados por conexões fortes é $\varepsilon_{2}$. Assim, o modelo incorpora um aspecto estocástico com relação também ao número de interações a cada iteração e à influência da reputação na adoção da tecnologia. 
Finalmente, a probabilidade de um indivíduo ser contaminado por causa dos esforços de marketing, das tendências de mercado e das conexões fracas e fortes da rede social é dada por:

ProbINFEC $(\mathrm{i})=$ 1 - (1 -ProbMARK(i)).(1 -ProbTREND(i)).(1 -ProbWEAK(i)).(1 -ProbSTRONG(i))

Nas simulações, para cada indivíduo sadio é obtida uma realização de uma variável aleatória com distribuição uniforme entre o e I. Caso essa realização da variavel aleatória seja menor que ProbINFEC(i), então o indivíduo é infectado, passando a adotar a tecnologia.

\section{RESULTADOS E CONSIDERAÇÕES FINAIS}

Inicialmente, vamos utilizar um caso-base para as simulações, cujos valores arbitrários dos parâmetros estão na Tabela I. Para fins de análise da performance do algoritmo desenvolvido em VBA para o Excel, cada simulação, com duzentas iterações, realizada em um Intel Celeron de 2,9 GHz, com 504 MB de RAM, leva cerca de 5 minutos.

\section{TABELA I}

PARÂMETROS DO MODELO TEÓRICO PARA O CASO-BASE

\begin{tabular}{ccccccccccc}
\hline PARÂMETRO & $N$ & $\rho$ & $\alpha_{1}$ & $\alpha_{2}$ & $\beta$ & $\gamma$ & $\delta_{1}$ & $\delta_{2}$ & $\varepsilon_{1}$ & $\varepsilon_{2}$ \\
\hline Valor & 100 & 0 & 0,4 & 0,9 & 0,02 & 0,001 & 0,02 & 0,08 & 0,05 & 0,1 \\
\hline
\end{tabular}

Os resultados de cinqüenta simulações para os parâmetros da Tabela I, bem como o cenário médio, são apresentados na Figura 4. Observe que a adoção da tecnologia pelos consumidores possui uma forma côncava em todos os estágios da difusão, denotando que a velocidade de evolução da tecnologia é positiva porém decrescente com o número de iterações. Simulações adicionais realizadas sugerem que a correlação $\rho$ entre os perfis de cultura e de gosto tem pouca influência no processo de difusão da tecnologia. 


\section{FIgURA 4}

\section{EVOLUÇÃO DA PROPORÇÃO DE USUÁRIOS DA TECNOLOGIA PARA O CASO-BASE}

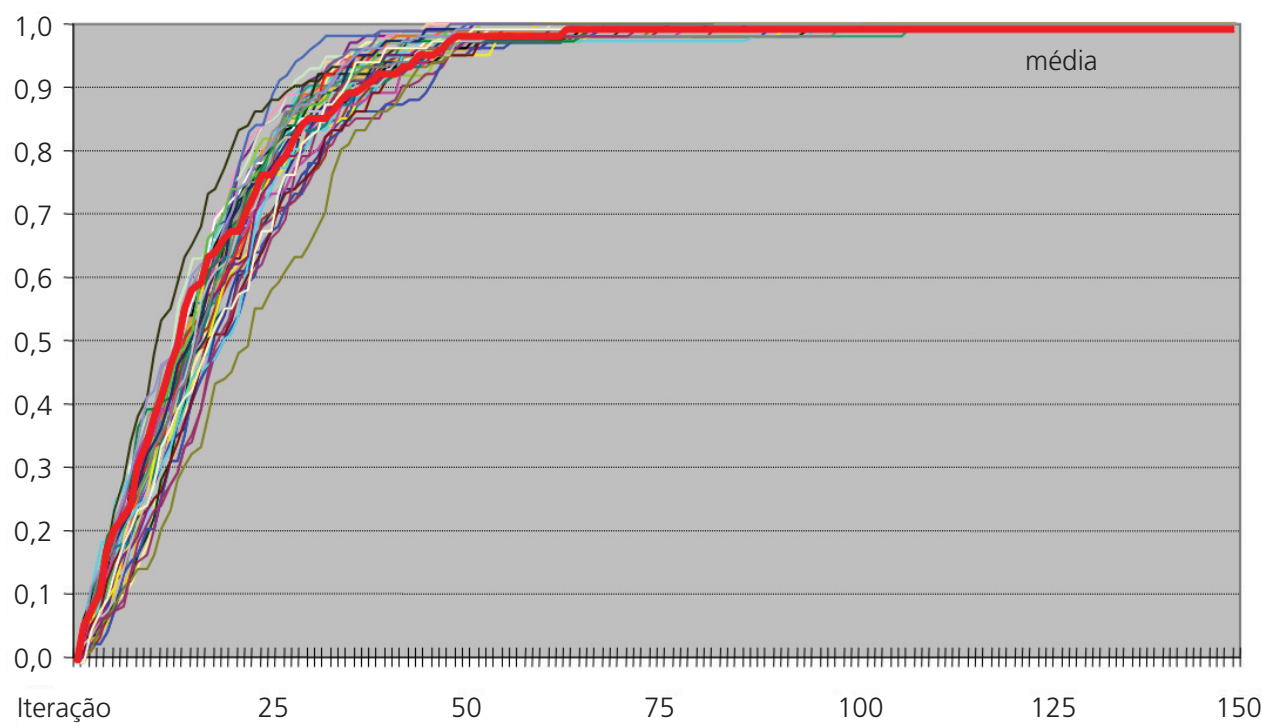

Com base na Figura 4, pode-se identificar o comportamento da evolução do uso da tecnologia. Porém, o modelo se torna mais útil quando são realizadas análises de sensibilidade, por meio da simulação de diferentes valores para os parâmetros. Neste caso, variações pequenas e sucessivas permitem a identificação de comportamentos não-lineares. Em uma primeira análise, é importante investigar o processo de difusão quando as redes sociais são mais ou menos densas, ou seja, quando existe um maior número de ligações ou conexões entre os indivíduos. Na Figura 5, são mostrados resultados específicos das topologias geradas em simulações escolhidas arbitrariamente, para ilustrar a estrutura e densidade das redes sociais. Os pontos representam os indivíduos; e os segmentos de retas, as conexões. Para gerar a topologia, utilizou-se o software NetDraw. 


\section{FIgURA 5}

\section{EXEMPLO DE TOPOLOGIAS DAS REDES,} EM FUNÇÃO DO NÚMERO DE CONEXÕES
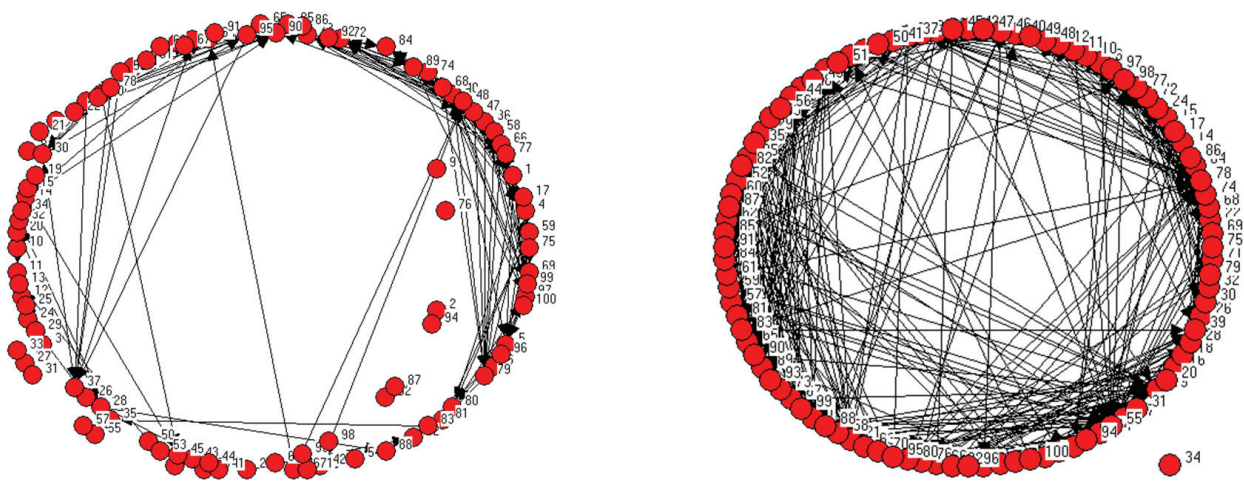

Observe que, de acordo com a Figura 2, quanto maior $\alpha_{\mathrm{I}}$, sendo fixo $\alpha_{2}$, menor a possibilidade de ocorrência de vínculos fracos. Quanto menor $\alpha_{2}$, com $\alpha_{\mathrm{I}}$ constante, maior a probabilidade de ocorrência de vínculos fortes e menor a probabilidade de ocorrência de vínculos fracos. A Figura 6 ilustra o resultado da simulação para situações de maior ou menor probabilidade de existência de vínculos fracos e fortes.

\section{FIgURA 6}

SIMULAÇÃO DA PROPORÇÃO DE USUÁRIOS EM FUNÇÃO DA QUANTIDADE DE CONEXÕES FORTES OU FRACAS

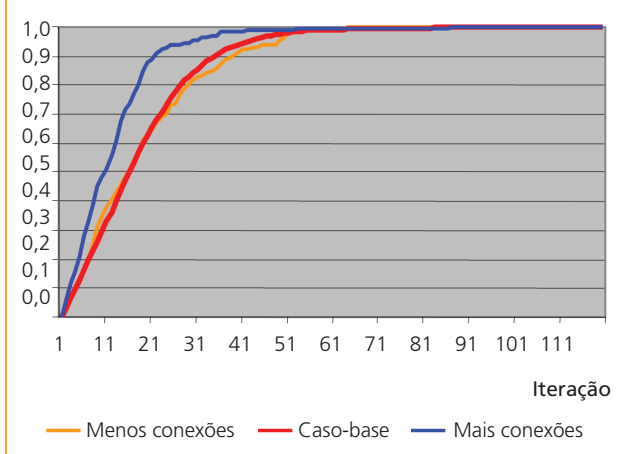

(a) Conexões fortes

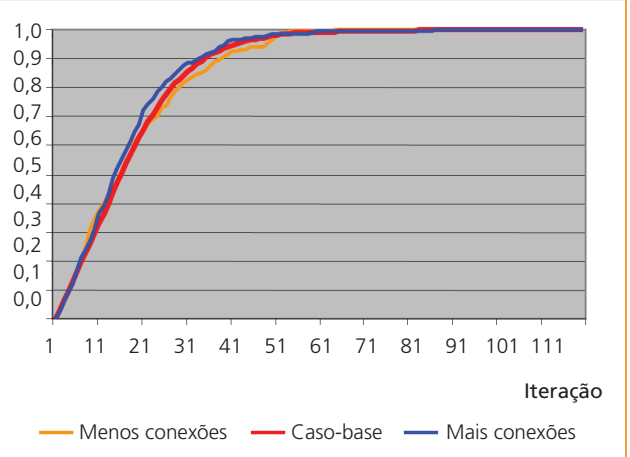

(b) Conexões fracas 
Identifica-se que os vínculos fortes, apesar de ocorrerem com uma freqüência menor, possuem uma maior influência na difusão da tecnologia quando comparados com os vínculos fracos. Essa evidência, apesar de parecer lógica, contraria, de certa maneira, os resultados de Granovetter (I973), que estabelecem a relevância das conexões fracas. Nas simulações, o parâmetro associado ao número de conexões fracas não apresentou grande impacto na evolução do uso da tecnologia. Esse fato pode sugerir que, dado o grau de incerteza da nova tecnologia, os indivíduos tendem a se apoiar mais na rede de relacionamento mais próxima, denotada por ligações fortes. Essa análise estaria de acordo com Walker (I995), que identifica a importância das opiniões de familares e amigos na escolha de médicos ou advogados, conforme apresentado no "Referencial teórico".

Variando os parâmetros $\beta$ e $\gamma$, podemos estudar a influência dos fatores externos à rede social na evolução da proporção de usuários. Os resultados das simulações são descritas na Figura 7.

\section{FIGURA 7}

PROPORÇÃO DE USUÁRIOS EM FUNÇÃO DE FATORES EXTERNOS AO LONGO DAS ITERAÇÕES

(a) Marketing convencional $(\beta)$

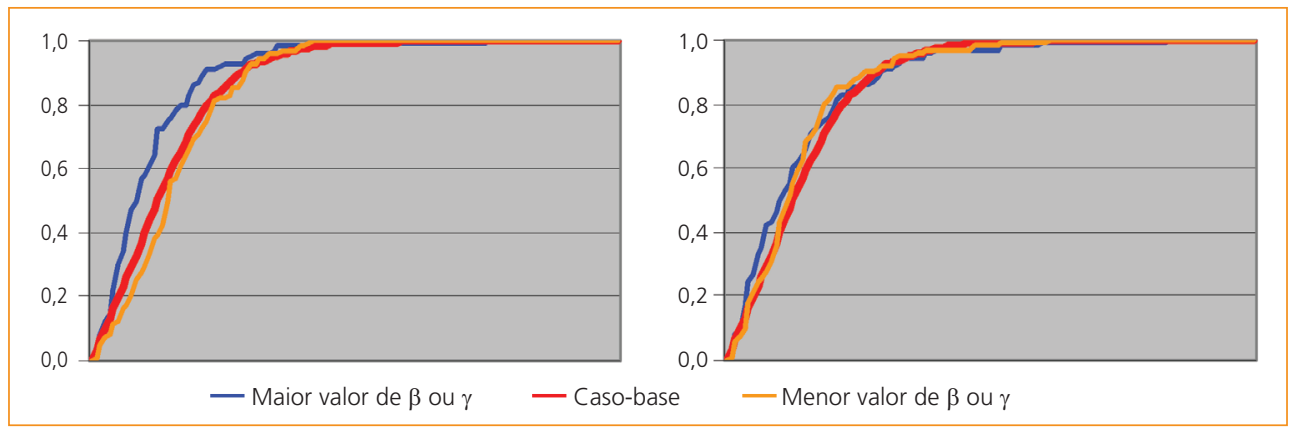

Destaca-se que o modelo privilegia, para o aumento da velocidade de difusão da tecnologia, os esforços convencionais de marketing. Uma vez que, na primeira iteração, nenhum indivíduo é contaminado, a presença de propaganda ou divulgação do produto é importante para que o processo de difusão possa se iniciar. A tendência de mercado sofre maior influência do número de infectados nos estágios iniciais da difusão. Os resultados sugerem que, a partir de um certo momento, principalmente nos estágios finais, a inércia pode acabar causando a infecção dos indivíduos que ainda não adotam a tecnologia. Assim, nos estágios mais avançados da tecnologia, praticamente não são necessários maiores investimentos em marketing. 
Considerando os aspectos internos, pode-se analisar o comportamento do uso da tecnologia. Variando-se os parâmetros $\delta_{\mathrm{I}}$ e $\delta_{2}$, $\varepsilon_{\mathrm{I}}$ e $\varepsilon_{2}$, obtêm-se os resultados apresentados na Figura 8. No modelo, quanto maior $\delta_{\mathrm{I}}$ e $\delta_{2}$, maior a possibilidade de contaminação em interações por meio de vínculos fracos e fortes, respectivamente. Como era esperado, a maior probabilidade de contágio implica uma maior velocidade de adoção de tecnologias. Porém, observa-se, mais uma vez, que a influência é maior nos vínculos fortes.

Resultado semelhante ocorre quando se analisa a influência da probabilidade de interação $\varepsilon_{\mathrm{I}}$ e $\varepsilon_{2}$ em cada tipo de vínculo. O modelo estabelece que não necessariamente um indivíduo vai interagir, a cada iteração, com todos os indivíduos aos quais está conectado, fraca ou fortemente. Mais uma vez, a sensibilidade da evolução do uso da tecnologia é maior para alterações do parâmetro referente à probabilidade de interação nos vínculos fortes. Enfatiza-se um comportamento não-linear da evolução da proporção de usuários considerando os diferentes valores dos parâmetros.

$\mathrm{Na}$ discussão do modelo teórico, foram explicitadas as conceituações de cada um dos parâmetros $\alpha_{\mathrm{I}}, \alpha_{2}, \beta, \gamma, \delta_{\mathrm{I}}, \delta_{2}, \varepsilon_{\mathrm{I}}, \varepsilon_{2}$, e apresentaram-se as referências que suportam a incorporação de cada variável relevante como ProfCULT, ProfTASTE, WILLINGNESS, Reputation no modelo. Devem ser destacadas as limitações e as potencialidades da modelagem teórica apresentada. Em primeiro lugar, o modelo somente considera relevantes as variáveis e os parâmetros contemplados. Na prática, a difusão do uso de tecnologias pode sofrer influência de outros fatores não incorporados no modelo. Em contrapartida, o modelo teórico permite que influências específicas sejam analisadas, sem a existência de fatores espúrios ou de problemas de operacionalização prática das variáveis. 


\section{FIGURA 8}

\section{PROPORÇÃO DE USUÁRIOS EM FUNÇÃO DE FATORES INTERNOS AO LONGO DAS INTERAÇÕES}

(a) Probabilidade de infecção nos vínculos fracos $\left(\delta_{\mathrm{I}}\right)$

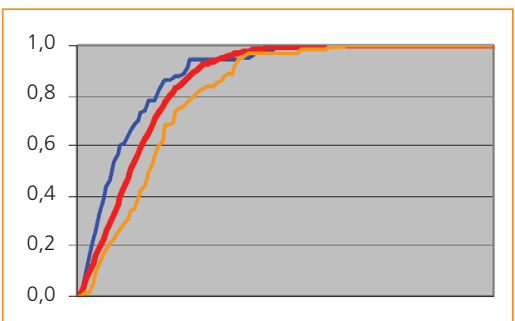

(c) Probabilidade de interação nos vínculos fracos $\left(\varepsilon_{\mathrm{I}}\right)$

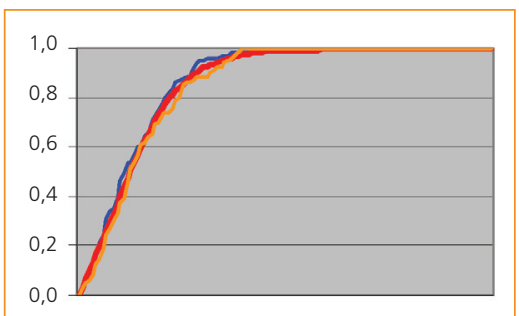

(b) Probabilidade de infecção nos vínculos fortes $\left(\delta_{2}\right)$

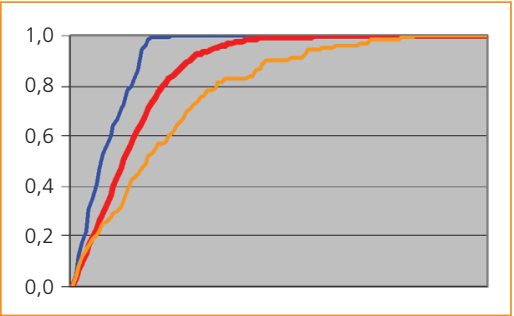

(d) Probabilidade de interação nos vínculos fortes $\left(\varepsilon_{2}\right)$

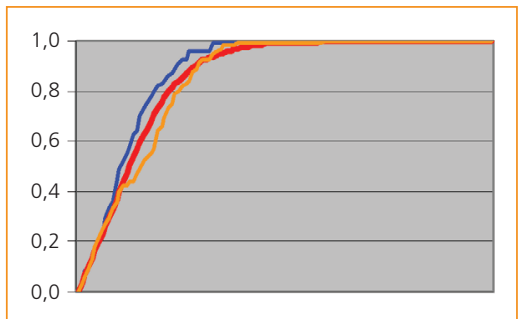

— Menor valor de $\delta_{1}, \delta_{2}, \varepsilon_{1}, \varepsilon_{2}$

Em segundo lugar, na simulação do modelo, a imposição de valores arbitrários para os parâmetros pode prejudicar a acurácia dos resultados, uma vez que não foi realizado um estudo empírico para estimação desses parâmetros. Porém, a análise de sensibilidade, com simulações usando diferentes valores para os parâmetros, pode levar a resultados comparativos que possibilitam a tomada de decisão para um maior aproveitamento dos esforços de marketing. Deve-se enfatizar que, para tecnologias novas, dificilmente é possível realizar uma estimação de parâmetros baseados em dados históricos, pois as condições iniciais podem ser bastante diferentes de valores atuais. Nesse contexto, o uso de parâmetros projetados e muitas vezes arbitrários pode auxiliar na formulação de estratégias por parte dos desenvolvedores de novas tecnologias.

Em terceiro lugar, o modelo teórico não sugere um mecanismo definitivo para a operacionalização de variáveis para uma pesquisa empírica. Porém, o modelo pode servir de base para o estabelecimento de instrumentos de mensuração dos parâmetros. Com a evolução das pesquisas na área, os referenciais 
teóricos e as evidências empíricas podem ser aprimorados, permitindo a construção de ferramentas de mensuração adequadas e a definição de constructos mais consistentes.

É importante destacar que os resultados do modelo não somente permitem uma avaliação das complexas relações, muitas vezes não-lineares, entre as várias variáveis envolvidas no processo de difusão de tecnologias, como também possibilitam a avaliação de cenários extremos e cenários mais prováveis. Dessa forma, pode-se obter um intervalo de confiança para a propagação da tecnologia para diferentes números de iterações do modelo. Os resultados do trabalho podem embasar a formulação de estratégias. Por exemplo, os resultados da simulação mostram que, para acelerar o processo de difusão de uma tecnologia, as estratégias mais indicadas seriam voltadas para o aumento dos parâmetros associados aos vínculos fortes. De forma menos contundente, aumentos nos parâmetros associados aos vínculos fracos também representam estratégias que conduzem a uma maior velocidade de adoção de tecnologias.

Um ponto que parece ser relevante envolve o grau de incerteza da tecnologia. Provavelmente, inovações mais relevantes ou que envolvem um nível de incerteza maior, com impactos potenciais maiores, sofrem maior influência das interações por meio dos vínculos fortes. Em contrapartida, é provável que inovações com menos incerteza, que causem um menor impacto em razão de menores custos de implementação e de mudança, sofram também influência relevante das interações por meio dos vínculos fracos. Essa é uma hipótese que poderia ser analisada em estudos futuros.

Em termos práticos, o modelo deste artigo pode sugerir algumas estratégias. Por exemplo, a velocidade de difusão poderia ser incrementada por meio de um maior esforço de marketing convencional nos estágios iniciais da introdução da tecnologia. Em estágios intermediários, os esforços poderiam ser direcionados para a propagação de informação por vínculos das redes sociais, tendo em vista a relevância das conexões. De certa maneira, algumas empresas já adotam estratégia análoga. Exemplificando, ao darem descontos a indivíduos de uma mesma família que usam os serviços de uma mesma operadora, as empresas de telefonia buscam propagar sua tecnologia por meio de vínculos fortes.

Considerando os novos meios de comunicação e interação e a relevância dos vínculos das redes sociais, uma maneira de aumentar a velocidade de difusão de tecnologias seria fomentar uma maior aproximação dos indivíduos. Assim, a maior força dos vínculos causa uma maior influência no processo de difusão. Nesse contexto, a exploração, para fins mercadológicos, de ferramentas como o Orkut, parece ser uma estratégia viável e até mesmo previsível. Dessa forma, meios de comunicação que proporcionem forte interação entre usuários podem constituir um novo campo de batalha para empresas de marketing. 


\section{REFERÊENCIAS}

ARMENGOL, A.; JACKSON, M. The effects of social networks on employment and inequality. The American Economic Review, v. 94, n. 3, p. 426-462, 2004.

ARDNT, J. Role of product-related conversations in diffusion of a new product. Journal of Marketing Research, v. 4, n. 3, p. 29I-295, I967.

AXELROD, R. The complexity of cooperation: agent-based models of competition and collaboration. Princeton: Princeton University Press, I997.

BARTON, H.; MCMANUS, B. Technology diffusion and market structure: evidence from infertility treatment markets. Working paper, John M. Olin School of Business, September 2005.

BASS, F. A new product growth model for consumer durables. Management Science, v. I5, p. 2I5227, 1969 .

BOURDIEU, P. Distinction: a social critique of the judgment of taste. London: Routledge \& Kegan Paul, I984.

BRASS, D. Being in the right place: a structural analysis of individual influence in an organization. Administrative Science Quarter, v. 28, p. 518-529, I984.

BUSKENS, V. Social networks and the effect of reputation on cooperation. In: 6TH INTERNATIONAL CONFERENCE ON SOCIAL DILEMMAS. Proceedings... Wassenaar, I998.

BUTTLE, F. Word-of-mouth: understanding and managing referral marketing. Journal of Strategic Marketing, v. 6, p. 242-254, 1998.

COLEMAN, J. Social capital in the creation of human capital. American Journal of Sociology, v. 94, p. 95-I20, I988.

COLLIER, P. Social capital and poverty. Washington, DC: The World Bank, I998.

CUMMINGS, J. Work groups, structural diversity, and knowledge sharing in a global organization. Management Science, v. 50, n. 3, p. 352-383, 2004.

ELIASHBERG, J.; JONKER, J-H.; SAWNEY, M.; WIERENGA, B. Moviemod: an implementable decision-support system for prerelease market evaluation of motion pictures. Marketing Science, v. I9, n. 3, p. 226-243, 2000.

FOMBRUN, C. J. Strategies for network research in organizations. Academy of Management Review, v. 7, n. 280-29I, I982.

GRANOVETTER, M. The strength of weak ties. American Journal of Sociology, v. 78, p. 1360-1380, I973.

GOLDENBERG, J. L., B.; MULLER, E. Talk of the network: a complex system look at the underlying process of word-of-mouth. Marketing Letters, v. I2, n. 3. p. 2II-223, $200 \mathrm{I}$.

HARRISON, J. R.; CARROLL, G. R. The dynamics of cultural influence networks. Computational and Mathematical Organization Theory, v. 8, n. I, p. 5-30, 2002.

HERBSLEB, J. D.; MOCKUS, A. An empirical study of speed and communication in globally distributed software development. IEEE Transactions on Software Engineering, v. 29, n. 6, p. 48I-494, 2003.

KEMPE, D.; KLEINBERG, J.; TARDOS, E. Influential nodes in a diffusion model for social networks. Proceedings... Lisboa, 2005.

KREPS, D.; WILSON, R. Reputation and imperfect information. Journal of Economic Theory, v. 27, p. 253-279, I982. 
KRIDER, R.; WEINBERG, C. Competitive dynamics and the introduction of new products: the motion picture timing game. Journal of Marketing Research, v. 35, n. I, p. I-I5, I998.

KROEBER, A.; KLUCKHOHN, C. Culture. New York: Meridian Books, I952.

LEENDERS, R.; ENGELEN, J.; KRATZER, J. Virtuality, communication, and new product team creativity: a social network perspective. Journal of Engineering and Technology Management, v. 20, p. 69-92, 2003.

LIN, Z.; LI, D.; HUANG, W. Modeling trader reputation distribution in an online $\mathrm{C}_{2} \mathrm{C}$ auction market. IN: IRMA INTERNATIONAL CONFERENCE. Proceedings... Seatle, 2002.

LIU, H.; MAES. P.; DAVENPORT, G. Unraveling the Taste Fabric of Social Networks. The Media Laboratory. Massachusetts: Massachusetts Institute of Technology, 2005. (Working paper).

MILGRAM, S. The small world problem. Psychology Today, v. 22, p. 6I-67, I967.

MURPHY, K. M.; SHLEIFER, A. Persuasion in politics. The American Economic Review, v. 94, n. 2, p. 435-446, 2004.

OTTE, E.; ROUSSEAU, R. Social network analysis: a powerful strategy, also for the information sciences. Journal of Information Science, v. 28, n. 6, p. 44I-453, 2002.

OZCAN, K. Modeling word-of-mouth and consumer choice in the network society. Working paper. University of Michigan Business School, 200I.

PARK, R.; BURGESS, E. An introduction to the science of sociology. Chicago: University of Chicago Press, I92I.

PODOLNY, J. M.; BARON, J. N. Resources and relationships: social networks and mobility in the workplace. American Sociological Review, v. 62, p. 673-693, I997.

PUTNAM, R. The prosperous community-social capital and public life. American Prospect, v. I3, p. 35-42, I993.

RAUB, W.; WEESIE, J. Reputation and efficiency in social interactions: an example of network effects. American Journal of Sociology, n. 96, p. 626-654, I990.

RAUCH, J. E. Business and social networks in international trade. Journal of Economic Literature, v. 39, n. 4, p. II77-I203, 2001.

RESNICK, P.; ZECKHAUSER, R. Trust among strangers in internet transactions: empirical analysis of eBay's Reputation System. NBER, 200I. (Working paper).

ROGERS, E. Diffusion of innovations. New York: The Free Press, I962.

Diffusion of innovations. New York: The Free Press, I995.

SILVERMAN, G. How to harness the awesome power of word-of-mouth. Direct Marketing, p. 32-37, I997.

WALKER, C. Complexity. Los Angeles: Touchstone Books, I995.

WEJNERT, B. Integrating models of diffusion of innovations: a conceptual framework. Annual Review of Sociology, v. 28, p. 297-326, 2002.

\section{TRAMITAÇÃO}

Recebido em 28/8/2006

Aprovado em 3/1/2007 